\title{
Publisher Correction to: delivery systems of CRISPR/Cas9-based cancer gene therapy
}

Alessio Biagioni", Anna Laurenzana, Francesca Margheri, Anastasia Chillà, Gabriella Fibbi and Mario Del Rosso

Correction to: J Biol Eng (2018) 12: 33.

https://doi.org/10.1186/s13036-018-0127-2

In the original version of this article [1], published on 18 December 2018, there was 1 incorrect author name.

The incorrect author name was published as:

Mario so.

The correct author name is:

Mario Del Rosso.

The original publication of this article has been corrected.

The publisher apologizes to the authors and readers for the error and the delay in publishing this correction article.

Received: 14 January 2019 Accepted: 15 April 2019

Published online: 02 May 2019

\section{Reference}

1. Biagioni A, Laurenzana A, Margheri F, et al. Delivery systems of CRISPR/Cas9-

based cancer gene therapy. J Biol Eng. 2018;12(33). https://doi.org/10.1186/

s13036-018-0127-2.

* Correspondence: alessio.biagioni@unifi.it

Department of Experimental and Clinical Biomedical Sciences, University of

Florence, Viale G.B. Morgagni 50, 50134 Florence, Italy 